\title{
Self-determined distribution of local energy resources for ensuring power supply during outages
}

\author{
Selma Čaušević ${ }^{*}$ (D), Martijn Warnier and Frances M.T. Brazier
}

\author{
*Correspondence: \\ s.causevic@tudelft.nl \\ Systems Engineering Section, \\ Faculty of Technology, Policy and \\ Management, Delft University of \\ Technology, Jaffalaan 5, 2628 \\ BX,Delft, Netherlands
}

\begin{abstract}
Ensuring access to reliable and sustainable power supply is becoming more and more challenging due to a combination of factors such as more frequent power grid outages caused by extreme weather events, the large-scale introduction of renewable energy resources that increases the complexity of the power system, but also aging infrastructure, supply and demand imbalance and power theft in some areas. Combined, all these factors can cause outages and together they can make electricity supply unreliable. The implications of this are many, ranging from minor inconveniences to major failures of critical infrastructures. A potential solution to ensure power supply during outages is to use local generation in the form of renewable resources to supply energy. This paper proposes a community-based mechanism that demonstrates that when community members can determine for themselves how excess energy generation is distributed, the power supply of specific members can be ensured. Self-determination is achieved by prioritizing and differentiating between community members as well as automatically and continuously redistributing energy, thereby adapting to sudden changes in supply and demand. Simulation results show that the proposed mechanism can be used to empower local communities to decide for themselves how local resources are distributed during events such as outages, ensuring prolonged power supply for differentiated members of affected communities. Harnessing the potential of renewable resources and smart technologies for intelligent coordination through empowerment of consumers to become pro-active participants is a promising solution for the future power systems.
\end{abstract}

Keywords: Energy communities, Self-determination, Self-organization, Prioritization, Power outages, Local generation

\section{Introduction}

Power outages present a challenge for current and future power systems. Such outages can be caused by a number of factors including the increasing frequency and severity of extreme weather events due to climate change, infrastructure failures and aging power plants that require frequent maintenance, but also supply and demand imbalance. Power outages adversely impact people and other infrastructures that rely on electricity. The negative social impact of outages can be very severe, ranging from inconveniences to normal daily operations to failures of critical infrastructures such as drinking water and communication systems (Jiménez-Estévez et al. 2017). This challenge is further amplified

(c) The Author(s). 2019 Open Access This article is distributed under the terms of the Creative Commons Attribution 4.0 International License (http://creativecommons.org/licenses/by/4.0/), which permits unrestricted use, distribution, and reproduction in any medium, provided you give appropriate credit to the original author(s) and the source, provide a link to the Creative Commons license, and indicate if changes were made. 
by the rapid introduction of renewable energy resources. As these renewables are volatile \& non-dispatchable, they add uncertainty to and increase the complexity of an already highly complex power system.

At the same time, renewable resources owned by local communities provide a potential to ensure reliable power supply when the backbone grid is unavailable (Ilic et al. 2010; Palensky et al. 2014). Decentralization of energy systems through such smallerscale initiatives that rely on local resources is a promising approach for the future of smart grids (Panteli and Mancarella 2015; Wollenberg 2005). Existing solutions that deal with decentralization of power systems are associated with concepts such as microgrids and virtual power plants (VPPs), that aim to enable easier integration of distributed renewable resources and to empower consumers and prosumers (Hatziargyriou et al. 2007; Asmus 2010). VPPs are typically virtual groups of several types of energy resources for which the main motivation for grouping is usually shared access to an energy market for economic reasons (Asmus 2010). As such, their purpose is not to operate independently during outages. In contrast, microgrids are subsets of power systems that are planned to operate both in parallel with the main grid and independent of it in an islanded mode when needed. To enable this type of independent operation, they require sophisticated physical infrastructure and are as such tied to specific geographic locations (Asmus 2010; Panteli and Mancarella 2015). During outages a microgrid can be decoupled from the main grid and run in a stand-alone islanded mode which ensures continuous power supply within the microgrid. In principal, the rest of the power grid does not benefit from this behavior. The members of a microgrid agree to the terms and conditions of membership, and together form the ownership structure of a microgrid. Real-time adaptation of a membership is not common, if not impossible. This poses a constraint in case of unexpected events such as outages, where changes in supply and demand can vary in near real-time and mechanisms for dynamic supply and demand matching in changing groups of consumers and prosumers might be required (Strbac et al. 2015). During outages, multiple isolated microgrids can be connected to form a networked microgrid cluster and gain additional flexibility in outage response (Li et al. 2017). To harness the potential of multiple microgrids while still preserving their autonomy, a hierarchical outage management scheme (OMS) is proposed in Farzin et al. (2016) to enhance resilience of distribution systems during outages.

Community microgrids such as those in Japan (Sheldrick and Tsukimori 2017), supported by Japan's "National Resilience Program", and Brooklyn (Brooklyn Microgrid 2019), are a move towards more self-reliant, resilient power systems that ensure longterm, locally generated power supply within a community. To fully use the potential of these microgrids, active participation of all stakeholders from local communities is needed (Jiménez-Estévez et al. 2017; Taha and Panchal 2014).

Even though microgrids can deal with uncontrollable disturbances in an islanded mode, they can collapse under extreme, high impact events, such as floods, earthquakes etc. To be able to account for this type of events, a reliability and resilience framework can be applied, so that a microgrid can withstand social, technical, economic, or natural hazards without losing its functionality. A potential solution to quickly recover is by prioritizing which loads to connect if full recovery is not possible due to infrastructure damage (Jiménez-Estévez et al. 2017). Increasing self-sustainability of microgrids through load 
prioritization is considered in (Silva et al. 2015), where multi-agent systems (MAS) are used to represent members of a microgrid as intelligent agents that operate autonomously to achieve local goals. In this paper, prioritization is performed on the level of consumers that prioritize their own controllable loads by applying shedding and re-scheduling procedures (Silva et al. 2015). Consumer prioritization with the purpose of consumer-centric energy management is proposed in (Tushar et al. 2014).

Load prioritization is routinely used for load shedding, curtailment, system restoration and microgrid management (Reddy et al. 2017; Mondal et al. 2016; Gao et al. 2016). Different types of loads are considered, such as critical and controllable loads (Huang et al. 2016). With respect to outage management, when restoring loads, utilities currently prioritize them based on their importance for general concepts such as public safety and health. However, this process is static and does not take into account neither real-time conditions of an outage, nor the specifics of a community an outage occurs in (Arghandeh et al. 2014). To bring in the societal perspective with respect to load prioritization during outages, systems such as Styrel in Sweden have been introduced, where consumers are categorized into pre-defined categories and assigned classes and points, based on which electricity distribution is performed during a power shortage (Landegren et al. 2014; Grosse and Olausson 2018). However, this type of prioritization still does not include active participation of communities affected by outages that can be empowered to make local decisions regarding their energy resources. Thus, different prioritization schemes are needed to reflect local needs of an affected community and real-time conditions of a specific outage (Arghandeh et al. 2014).

Methodologies for active engagement of communities in the introduction and management of local energy resources facilitate sustainable resource management in future power systems (Alvial-Palavicino et al. 2011; Cai et al. 2017; Jung et al. 2016; Adu-Kankam and L. M. -Matos 2018; Katre et al. 2019; Arentsen and Bellekom 2014; Hufen and Koppenjan 2015). Energy communities can potentially play an important role in facilitating energy interventions, because they enhance trust relationships, support behavioral changes and encourage other local benefits, such as lower energy bills, new local jobs, increase in the sense of ownership, but also reduction of carbon emissions (OVO Energy 2014; Šćepanović et al. 2017). As traditional, centralized power systems transform into more decentralized, prosumers and consumers acquire a more active role in decision making on the local level (Silva et al. 2015; Oteman et al. 2014). Such active engagement of local communities increases social acceptance (Jiménez-Estévez et al. 2017) and can significantly enhance the reliability of power supply (Tushar et al. 2014).

To address the challenges related to intelligent coordination of an increased number of local renewable resources and empower consumers to become pro-active participants of the future energy systems, new paradigms for power system coordination and planning will be needed (Ilic et al. 2010; Palensky et al. 2014). A potential solution that ensures (partial) continuation of power supply during outages for communities in frequently affected areas, is to rely on locally generated supply in the form of renewables. To this purpose communities need to agree to share energy, as an energy community. As such, they can use local resources to their full capacity, and collectively decide how to distribute energy locally.

This paper bridges the concepts of local engagement from energy communities with features such as islanding from existing decentralization mechanisms (microgrids), and 
builds upon them to ensure prolonged power supply in areas frequently affected by outages. The concepts are further extended by allowing communities to dynamically adapt to changes in their environment. This paper assumes that although a limited number of local renewable resources is available, they will not always be sufficient to meet the demand of all community members affected by an outage. Due to scarcity of available resources, a decision on how to distribute these resources has to be made. In contrast to existing outage management techniques where loads are prioritized before an outage occurs by a central authority, this paper brings in the community perspective by letting members of communities prioritize consumers and prosumers themselves and, using that prioritization, distribute local resources in a fully decentralized manner. For this purpose, this paper proposes a mechanism that supports communities affected by an outage to determine for themselves (self-determine) how local energy resources are distributed. It does so by differentiating between consumers and prosumers in the area. For example, a community can decide that schools should have electricity during an outage, making the school a primary destination for locally produced electricity. Or a community can decide to allocate local energy resources to a sports field if an outage occurs during a match. In both cases social arguments are the basis for such decisions.

The main contribution of this paper is twofold. One, it demonstrates that by empowering members of communities to decide for themselves how local resources are distributed during an outage, the duration of power supply can be prolonged for specific members of affected communities; and two, it proposes and develops a fully decentralized mechanism serving as an ICT platform for energy sharing in communities frequently affected by outages.

The rest of the paper is organized as follows: "Self-determined distribution of local resources" section describes the concepts of the proposed mechanism, "System setup" section describes the system, assumptions and the mechanism's algorithm in detail, and "Experiments" section describes the experiments conducted, as well as the key performance indicators used to assess the mechanism outputs and the experimental setup used. "Results" section presents the results, while "Discussion" section discusses potential applications, as well as the limitations of the proposed mechanism with respect to the assumptions made. Finally, "Conclusions" section concludes the paper.

\section{Self-determined distribution of local resources}

This section describes the main concepts of the proposed mechanism for self-determined distribution of local energy resources during outages, based on differentiation. As stated in "Introduction" section, the main objective of this paper is to demonstrate that by letting communities decide for themselves how local resources are distributed during an outage, the duration of power supply can be prolonged for specific members of affected communities. This objective is achieved by first demonstrating that supply and demand can be matched based on locally available information only, using a dynamic clustering sub-mechanism, and then by including prioritization as a means to differentiate between different consumers and prosumers. These two sub-mechanisms are used to form selforganized local energy communities that are self-sustainable during outages. "Dynamic clustering" and "Prioritization" subsections explain the two sub-mechanisms in detail, respectively. 


\section{Dynamic clustering}

This paper builds upon the mechanism proposed in (Čaušević et al. 2017) that enables decentralized supply and demand balancing in energy systems. Consumers, prosumers and producers of electricity rely on local knowledge of their production and/or demand to organize themselves (self-organize) into small-scale local energy communities (clusters) that locally match supply and demand (Amin 2001; Farhangi 2010; Amin and Stringer 2008; Brazier et al. 2015). The main objective of clusters is to minimize the supply and demand mismatch within the cluster. Clusters act as autonomous groups that enable energy sharing of local resources. They can dynamically reconfigure by changing their composition to adapt to changes in the (external) environment or their (internal) preferences (Čaušević et al. 2017). This subsection briefly describes the previously proposed mechanism.

Distributed multi-agent systems (MAS) are used to represent cluster members (e.g. households, schools and supermarkets) as intelligent agents. A dedicated simulation tool developed in the Java programming language is used to setup multi-agents and conduct experiments. The tool implements basic agent behavior and enables asynchronous message exchange and processing. The agents have perfect knowledge of their supply and/or demand, and can communicate with each other, exchanging information about their location, and load and production profiles. Using a distributed information exchange algorithm (gossiping), agents build their own local view of the neighborhood. A distributed approach reduces the risk of single point failure in the network, and can be applied to a variety of scenarios where full knowledge of the system is unavailable. After building their own local views, agents send cluster membership offers to neighboring agents that best match their load and/or production profile. As the main objective of power systems is to match supply and demand of electricity (Wood et al. 2013), the best match is that which minimizes the mismatch. The mismatch between an agent and a cluster at time $t$ is calculated using Eq. 1 for the entire period for which clustering is performed. Contrary to the previously published work in (Čaušević et al. 2017), the optimization function for calculating the mismatch is adapted in this paper. The best match is found using Eq. 2. For the clustering period, the number of hours with zero mismatch $\left(\mathrm{Z}_{h}\right)$, negative mismatch/underproduction $\left(\mathrm{UP}_{h}\right)$, and positive mismatch/overproduction $\left(\mathrm{OP}_{h}\right)$ is counted, and the total amount of underproduction $\left(\mathrm{UP}_{a m t}\right)$ and overproduction $\left(\mathrm{OP}_{a m t}\right)$ is calculated. Weights are assigned to every parameter $\left(\omega_{z h}, \omega_{\text {UPh }}, \omega_{\text {UPamt }}, \omega_{\text {OPh }}\right.$, and $\left.\omega_{\text {OPamt }}\right)$.

$$
\begin{aligned}
& \text { mismatch }(a, c)_{t}=\operatorname{Supply}(a, c)_{t}-\operatorname{Demand}(a, c)_{t} \\
& \min \frac{1}{\omega_{z h} Z_{h}}\left(\omega_{U P h} * U P_{h}+\omega_{\text {UPamt }} * U P_{a m t}+\right. \\
& \left.\omega_{\mathrm{OPh}} * O P_{h}+\omega_{\mathrm{OPamt}} * O P_{\text {amt }}\right)
\end{aligned}
$$

where,

$$
\omega_{z h} \gg \omega_{\text {UPh }} \gg \omega_{\text {UPamt }} \gg \omega_{\text {OPh }} \gg \omega_{\text {OPamt }}
$$

To join a cluster, agents negotiate cluster membership, and this negotiation results in service level agreements (SLAs). SLAs are used as a means of fixing cluster composition, specifying the terms of electricity provisioning (Clark 2014; Methenitis et al. 2018) and committing agents to those terms. Clusters are either fixed for a relatively long period 
of time, or dynamically reconfigure to respond to changes in supply and demand of the agents.

\section{Prioritization}

This paper introduces the concept of consumer and prosumer differentiation based on community preferences. To enable differentiation of consumers and prosumers, local communities agree on the levels of supply priority (high, medium, or low) and pre-assign them to consumers and prosumers, before an outage occurs. These priorities indicate, for example, the social importance of a consumer or prosumer. Some priorities may be fixed, such as, for example, high priority for a school or a supermarket, while other priorities can change depending on the specific situation. The mechanism through which this differentiation is performed is referred to as prioritization. Prioritization aims to ensure that electricity is supplied to the highest priority consumers and prosumers first, if there is enough supply to meet their demand. The remaining electricity is then distributed to those with lower priority.

The prioritization mechanism is used to allow local communities to assign levels of supply priority to individual consumers and prosumers, and to decide for themselves how locally available renewable resources are distributed, so that prolonged power supply during outages is ensured. In the rest of this paper, prolonged power supply during outages is referred to as reliable power supply.

Supply reliability depends on the amount of leftover supply in the system. Leftover supply is energy supply that is left after all of the prosumers have consumed the energy they have produced to meet their own demand. It also includes any other renewable production that is not generated by prosumers, such as independent wind turbine generation. Thus, leftover supply depends on the type of renewable resources in the system (e.g., if only solar is available, there is no production during the evening, or during cloudy periods). Each level of priority requires to be supplied for a minimum number of hours of leftover supply. For example, if the system total leftover supply is $10 \mathrm{~h}$, the highest priorities can be supplied for a minimum of e.g. $7 \mathrm{~h}$. This parameter is reconfigurable and can be changed at any time. It can be different for every priority (e.g. higher priorities can require more hours of supply, while the lower ones can settle for fewer), or even different for every agent. The required minimum number of hours of leftover supply is crucial for assessing supply reliability for every priority. If an agent is supplied for a minimum number of hours required by its priority, the agent is said to have reliable supply during the outage period.

\section{System setup}

This section describes the system used for implementation of the proposed mechanism, including the main assumptions on which it is based. Furthermore, a detailed algorithmic description of the mechanism is given.

\section{System assumptions}

This paper considers areas that are frequently affected by planned and unplanned outages, and have high, but limited penetration of distributed renewable resources, locally owned by members of affected communities. It is assumed that no non-renewable production such as that of diesel generators is available. In terms of sustainability, two types 
of community members are distinguished: self-sufficient and non-self-sufficient members. Self-sufficient members are those who can sustain themselves with their own resources for at least an hour during an outage. Consequently, non-self-sufficient members are those who do not have any production of their own, or not enough to meet their own demand. During an outage, prosumers first meet their own demand using their own generated supply. Then, supply that is left is distributed to the highest priorities first using the proposed mechanism. The term "priorities" in this context refers to all the consumers and prosumers of the same priority. This resource distribution approach results in formation of clusters, that dynamically reconfigure to adapt to changes in their environment and have as a purpose reliable supply provisioning and maximization of self-sufficiency.

This paper assumes that communities themselves pre-determine the priorities. Negotiation mechanisms for assigning priorities are outside the scope of this paper. Assuming that there are fewer consumers and prosumers who should have the highest priority, compared to the medium and low priorities, priorities are assigned randomly in such a way that there is a higher probability that an agent is low or medium priority, compared to the high priority.

The proposed clustering mechanism abstracts from the physical layer of power systems. This paper assumes that there is a physical infrastructure that connects all consumers, prosumers and resources in an area affected by an outage, so that electricity can be distributed from any one point to another. Additionally, this paper assumes that the infrastructure enables "closing" and "opening" of connections between consumers, prosumers and available resources, so that electricity can be distributed to selected consumers and prosumers. Clustering creates clusters for which the topology is not fixed nor restricted by geographic location, and relies on existing communication infrastructure to exchange information between agents to create the grouping. This paper assumes that agents have perfect knowledge of their supply and/or demand profiles for the duration of clustering period.

Finally, this paper assumesthat the area for which clustering is performed is part of a single distribution system that is affected by a planned or an unplanned outage. Consequently, there is no electricity supply from the backbone grid.

\section{Algorithm details}

As shown in Fig. 1, at the beginning, every consumer, prosumer and producer (e.g. a wind turbine) is represented by an intelligent agent (a piece of software) that has perfect knowledge of its load and/or production profile and geographic information. Thus, a consumer is represented by a consumer agent $(C A)$, a prosumer by a prosumer agent (PSA), and a producer by a producer agent (PDA). Additionally, CAs and PSAs are assigned a supply priority level, based on which, the decision of electricity distribution is made. PDAs do not have a priority level, as they only supply electricity. When an outage occurs, agents exchange information on their geographic location to build a local view of the neighborhood. Information exchange is performed using a distributed information exchange algorithm (gossiping), where every agent in the system is connected to every other agent, directly or via other agents (Jelasity et al. 2005; Duckham 2013). After building the local view of a neighborhood (which depends on the neighborhood size), the dynamic clustering phase begins. This phase is executed for every time period for which 


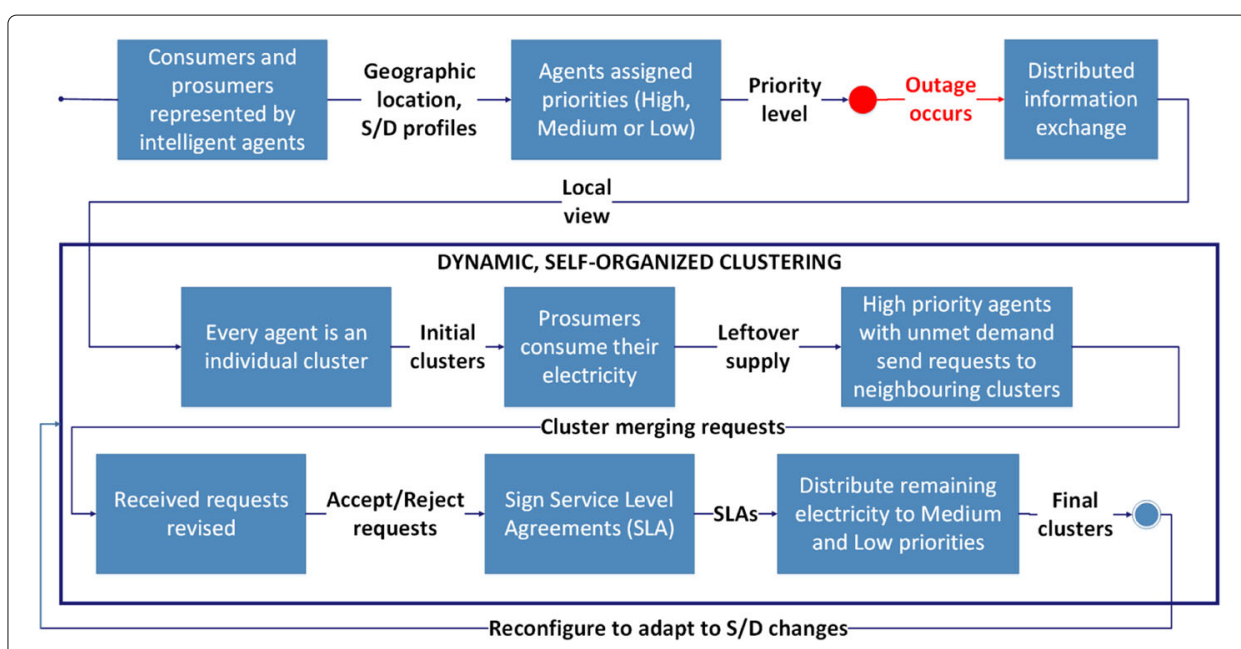

Fig. 1 Dynamic, priority-based clustering mechanism

clustering is performed (e.g. every hour or every day). Initially, each agent is a cluster on its own.

During an outage, each PSA first meets its own demand with the generated supply from, for example, solar panels. PSAs that have supply left after meeting their own demand can now distribute it to selected CAs and PSAs in their neighborhood whose supply is not met. Next, the dynamic distribution of resources begins. All agents with high priority whose supply is not met send requests to neighboring clusters that best match their profile. Recall from "Dynamic clustering" subsections that the best match is the one that generates the minimum mismatch, and is found using Eq. 2. After all requests are sent, clusters that have received membership requests revise them. If a cluster contains any agents with high priority, it accepts the request only if accepting it does not result in underproduction. If all agents are of lower priorities, a request with the best match is accepted. After acceptance, agents sign SLAs with clusters. SLAs contain information about a cluster membership request, and information about a cluster topology including: a list of cluster members, their load and production profiles, levels of supply priority and the minimum number of hours for which they would like to be supplied. Thus, SLAs are used as a means to fix cluster boundaries and ensure supply reliability. If at the end of this stage there is still supply left, the same procedure is repeated for medium and then low priorities. In the end, final clusters are formed and fixed for the clustering period. During the next clustering period, clusters dynamically reconfigure to adapt to changes in the environment (changes in supply and demand or in priorities).

\section{Experiments}

This section describes the experiments conducted to study the output of the proposed clustering mechanism. To achieve the main objective of this paper, the two sub-mechanisms described in "Dynamic clustering" subsections and "Prioritization" are used, and experiments are conducted accordingly (1) to explore if supply and demand can be matched in a decentralized way by studying supply and demand mismatch in the system and observing the effects of static vs. dynamic clustering, and (2) to explore how prioritization can be used to ensure prolonged power supply during outages. "Experimen- 
tal setup" subsection describes the experimental setup, while "Experiment set 1: Static vs. dynamic clustering" subsections and "Experiment set 2: Prioritization and reliability assessment" describe both experiments, respectively.

Note that the aim of the experiments is not to do an exhaustive investigation of the feasibility of the proposed mechanism under various circumstances. The large uncertainty in for example power generation, power demand, seasonal variations or the frequency of outages, prohibits this. It would be possible to do such an analysis for a specific location and time frame, provided that both generation and demand profiles are available, however this is outside the scope of the current paper. The main motivation for the experiments conducted here is to demonstrate that, under specific conditions, given adequate resources in an affected community, there is a working mechanism that can be used by the communities to ensure supply reliability by deciding how to share available resources.

\section{Experimental setup}

The experiments are run with 500 agents, and consider two different renewable resources: rooftop solar and wind production. The neighborhood size for the distributed information exchange algorithm is set to $40 \%$, meaning that every agent can form clusters with maximum $40 \%$ of the agents in the system. Out of 500 agents, $2.8 \%$ are high, $33.8 \%$ are medium, and $63.4 \%$ are low priority.

For illustration purposes and due to data availability, the data from the Netherlands is used to model load and production profiles. The daily load profile data is obtained from NEDU (NEDU - Verbruiksprofielen.), the Dutch Energy Data Exchange, and represents an average load profile of a household consumer. To add diversity in household profiles, the load profile data from (NEDU - Verbruiksprofielen.) is varied by maximum $\pm 20 \%$ for every consumer modeled. The data from July 1, 2015 is used in the experiments, as a representative day with high solar production to demonstrate the potential of the mechanism, given enough resources (Čaušević et al. 2017). It should be noted that the mechanism would not work on a cloudy, windless day, provided only solar and wind generation is available.

Experiments are first run with rooftop solar production only, where $40 \%$ of agents are prosumers with solar panels and $60 \%$ are consumers. Solar production is chosen as the only resource in this setup to demonstrate the output of the clustering mechanism in a dynamic environment, where electricity is not available during the night. Residential solar panels with standard dimensions of $1.651 \mathrm{~m} \times 0.99 \mathrm{~m}$ (Matasci 2017) (total area of $1.63 \mathrm{~m}^{2}$ ) are modeled. According to (Lemmens et al. 2014), average rooftop area available for installing solar panels per Dutch household is $\approx 33 \mathrm{~m}^{2}$, which amounts to maximum 20 residential solar panels for $100 \%$ rooftop usage. However, to account for different orientation of rooftops, only $30 \%-60 \%$ rooftop area is used. This means that prosumers are modeled with a minimum of 6 and a maximum of 12 solar panels per household.

Solar production is calculated as in (Patel 1999), using the Dutch solar irradiance data (Koninklijk Nederlands Meteorologisch Instituut.). The load and production profiles for this setup are shown in Fig. 2. The ratio between self-sufficient and non-self-sufficient agents is shown in Fig. 3. The Fig. 3 shows the ratio in percentages, while Fig. 3 show the absolute values.

To diversify the available resources, wind production is added, where a community- or intermediate-size $500 \mathrm{~kW}$ turbine is modeled (EWT). 500 agents are modeled, of which 


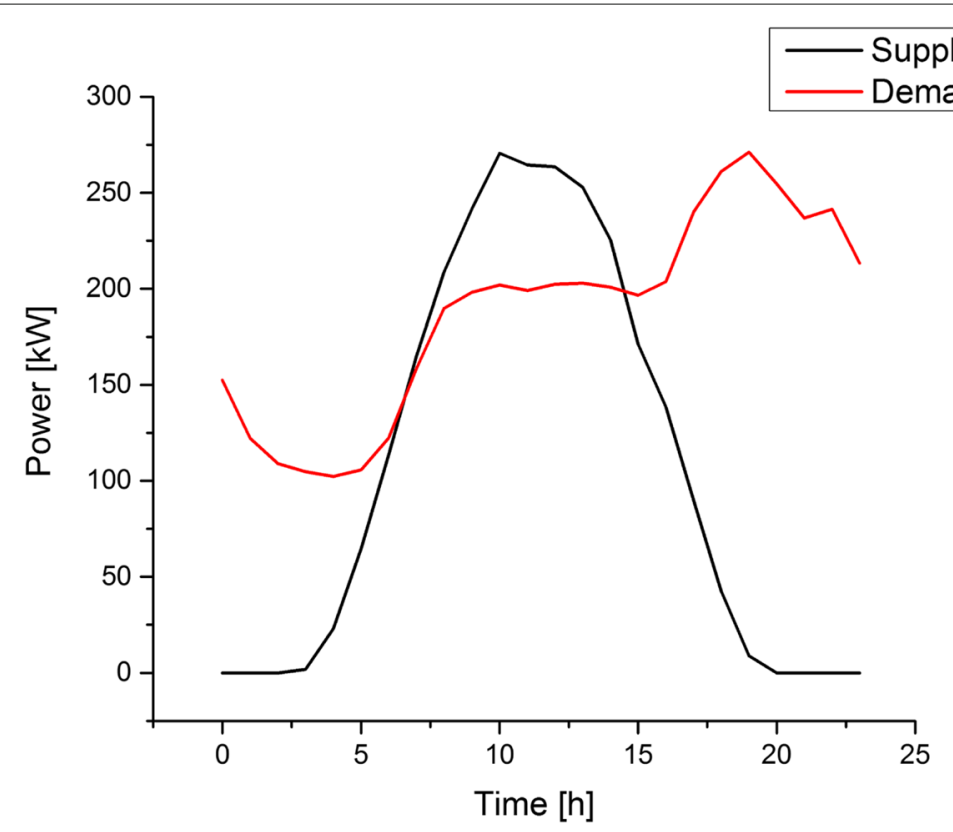

Fig. 2 Aggregate load and production profiles - rooftop solar production only

$40 \%$ are prosumers with solar panels, $0.2 \%$ wind turbines (thus, 1 wind turbine), and $59.8 \%$ consumers. In the rest of this paper, the wind turbine is referred to as a producer. Wind production is calculated as in (Patel 1999), using Dutch wind speed, temperature, and air pressure data (Koninklijk Nederlands Meteorologisch Instituut.). The load and production profiles for this setup are shown in Fig. 4.

It is important to note that all parameters set for the experiments are the best estimate for a given case scenario, as no data on a real case scenario is available. The proposed mechanism, as well as the experimental parameters observed, can serve as a starting point to address future challenges in such areas. However, thorough analysis of potential parameter settings in an area observed should be performed, and decisions should be made accordingly.

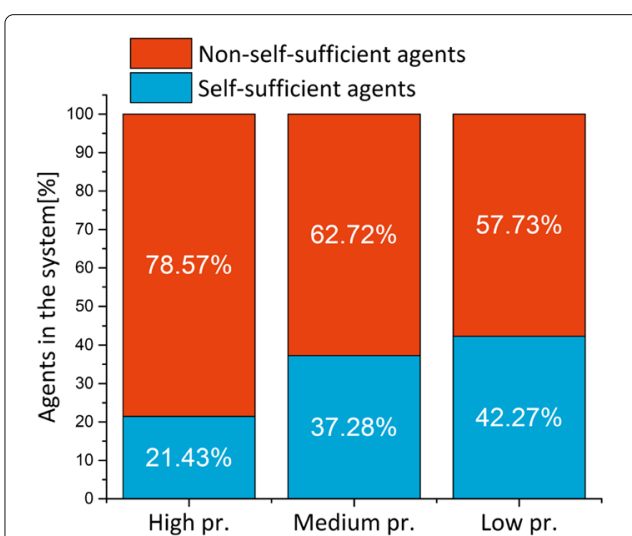

(a)

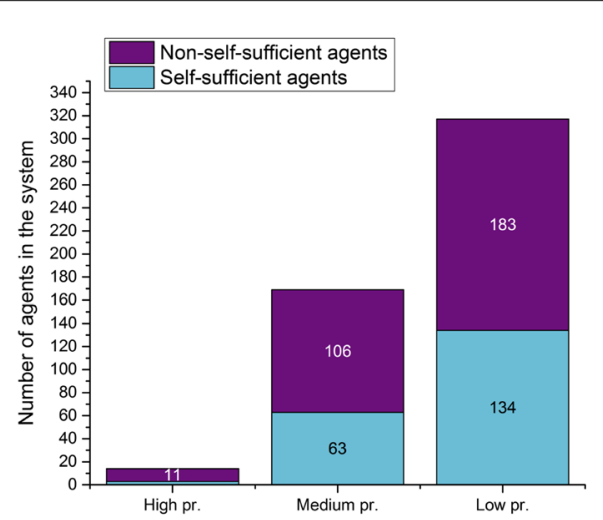

(b)

Fig. 3 Self-sufficient and non-self-sufficient agents in the system in $\mathbf{a}$ percentages and $\mathbf{b}$ absolute numbers 


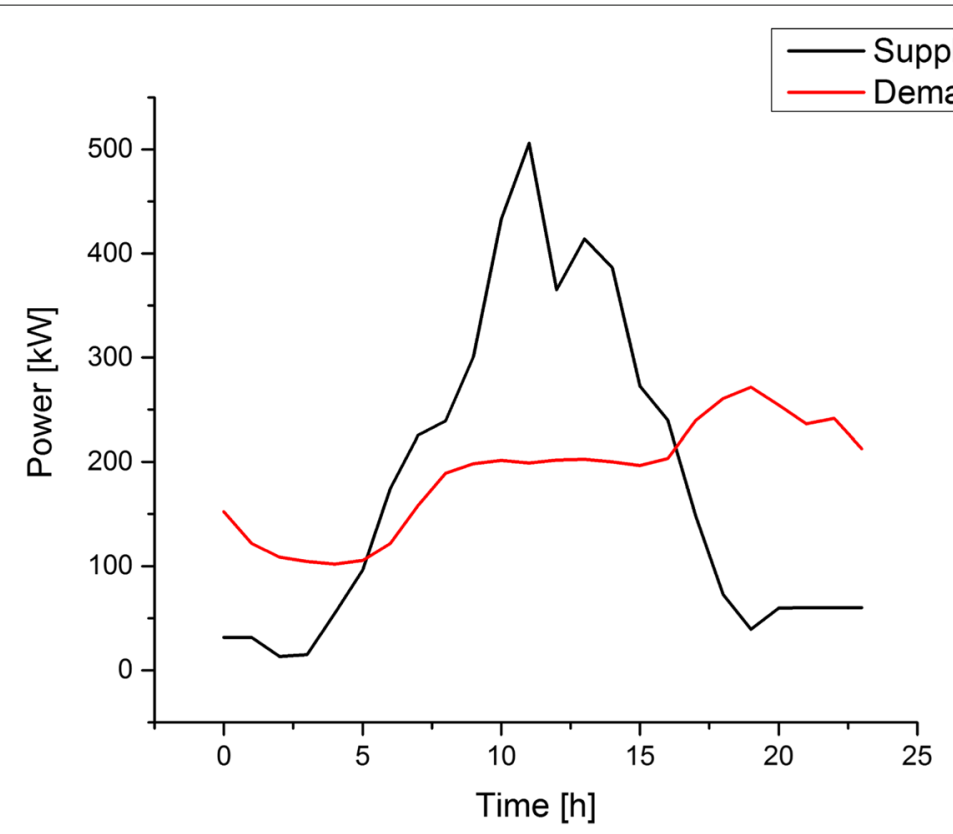

Fig. 4 Aggregate load and production profiles - wind and rooftop solar production

Experiment set 1: Static vs. dynamic clustering

To explore if supply and demand can be matched in a decentralized way with the same level of performance (minimum supply/demand mismatches) of a centralized system, and to observe the effects of changing the frequency of clustering reconfiguration, three clustering scenarios are used, namely: (a) daily clustering (static), (b) clustering every $8 \mathrm{~h}$ (dynamic), and (c) hourly clustering (dynamic). In (a), clusters are formed and fixed for a period of $24 \mathrm{~h}$, based on $24 \mathrm{~h}$ perfect forecast of agents' load and production profiles. In (b), clusters reconfigure every $8 \mathrm{~h}$, reflecting changes in the standard load profile of households. The most dynamic reconfiguration scenario is (c), where clusters reconfigure every hour to respond to changes in the environment. All three cases are compared to the centralized case, where no decentralized matching is performed and all agents are part of one big cluster. The centralized case is taken as the theoretical (and currently practical) best solution in terms of supply and demand balancing. Thus, the motivation is not to perform better than the centralized system, but to have a decentralized system that can operate almost as well as the centralized, giving control to individual agents.

For the purpose of these experiments, all agents are assumed to be part of a single distribution system. In this case, the distribution system considered is not affected by an outage. Consequently, there is no differentiation between consumers and prosumers. In these experiments, only rooftop solar production is considered as a locally available renewable resource. When there is surplus of electricity, it is fed into the backbone grid, while lack of supply is met by drawing electricity from the main grid.

The experiments are assessed using the following key performance indicators (KPIs):

1. Total overproduction (TOP) - this KPI looks at clusters that have more supply than needed, and calculates how much overproduction they generate in total (per hour)

2. Total underproduction (TUP) - this KPI looks at clusters that do not have enough supply, and calculates how much underproduction they generate in total (per hour) 
3. TOP deviation from centralized system (TOP DEV) - this KPI calculates how much the proposed clustering mechanism deviates from the centralized system in terms of total overproduction

4. TUP deviation from centralized system (TUP DEV) - this KPI calculates how much the proposed clustering mechanism deviates from the centralized system in terms of total underproduction

The main motivation is to minimize these KPIs for each clustering type (daily, $8 \mathrm{~h}$ and hourly). Table 1 gives an overview of all of the experiments conducted for dynamic vs. static clustering, as well as their setup.

\section{Experiment set 2: Prioritization and reliability assessment}

To explore how differentiation of consumers and prosumers using prioritization can ensure supply reliability for communities in areas affected by outages, priorities are assigned, and clustering is performed using the best-performing clustering scenario from "Experiment set 1: Static vs. dynamic clustering" subsection. In this experiment, the distribution system is considered to be affected by an outage, thus, there is no supply from the backbone grid.

Supply reliability is assessed with respect to the minimum required number of hours of supply. Three different case scenarios are considered:

1. In the first scenario, the priority level does not matter for the assessment of reliability. In this case, all agents with high, medium and low priorities need to be supplied during all, i.e. 100\%, of the hours with leftover supply in the system. This is an extreme case when no importance is given to the level of priority in terms of choosing reliability parameters, and no pre-assessment on leftover supply and the demand of agents is made. It can be seen as greediness of agents that are not willing to compromise and adjust to an outage situation.

2. In the second scenario, the priority level still does not matter for the assessment of reliability, but the minimum required hours of supply is set to a lower number. Now, all priorities need to be supplied during $90 \%$ of the hours with leftover supply. This minimum is set by the high priority agents, but since no difference is made between priorities in terms of parameter settings, other priorities require the same. In contrast with the previous case scenario, high priorities are willing to compromise and lower their requirement, since there might not be enough leftover supply for them all.

3. In the last scenario, a difference in terms of required number of hours of supply is made based on priority levels. Each level of priority needs to be supplied for a different minimum percentage of hours of leftover supply, with minimum set to: 90\% for high, 50\% for medium, and 30\% for low priorities. These numbers are chosen as the best estimate for different levels of supply priority and the system considered. The values can be set according to leftover supply in the system for every hour, and the demand of each of the priority level at those hours.

The three case scenarios are firstly run with only solar rooftop production as a locally available renewable resource. To diversify local production, and explore the effects of different renewable resources in the area, the third case scenario (with a difference between 


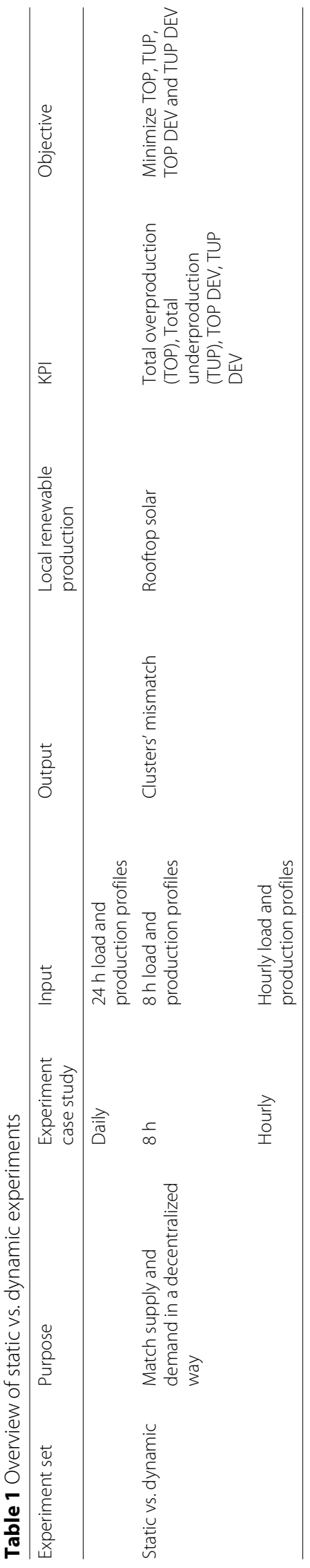


supply priority levels in terms of reliability requirement) is then run with both local solar and wind power.

All the experiments are assessed using the following KPIs:

1. Required supply reliability (RSR) - For each priority, the percentage of agents that meet the minimum required number of hours of supply. Agents do not require continuous supply, so the number of hours supplied is counted over the entire outage period, with or without hourly interruptions. This number denotes the number of hours supplied during an outage. It is compared to the minimum required by the level or priority, and the percentage of agents that met this minimum is calculated.

2. Self-sufficiency of clusters (SSC) - For each priority, the percentage of agents that are in $100 \%$ self-sufficient clusters. Self-sufficient clusters are those that have enough local resources to meet the demand of its members.

The main objective is to maximize these KPIs, in particular for high priority agents. Table 2 gives an overview of all of the experiments conducted for prioritization and reliability assessment, as well as their setup.

\section{Results}

This section presents the results obtained in the two main sets of experiments. First, a comparison is made between static and two types of dynamic clustering with the centralized approach in terms of total underproduction and overproduction. Then, the mechanism that performs the closest to the centralized approach is taken as the bestperforming, and is used in further experiments with prioritization to assess supply reliability during an outage. The following two Subsections discuss the results in detail.

\section{Experiment set 1: Static vs. dynamic clustering}

The results in this section present the ability of agents to match their supply and demand in a decentralized fashion based on local information only. Each of the case scenarios for static vs. dynamic clustering (see "Experiment set 1: Static vs. dynamic clustering" subsection) is considered in terms of total overproduction and total underproduction.

As shown in Fig. 5a and 5b, clustering every hour generates clusters that have overall lowest total underproduction and overproduction, and the system operates almost the same as the centralized system. Daily clustering and clustering every $8 \mathrm{~h}$ both follow a similar trend, but with more overproduction and underproduction compared to hourly clustering. Table 3 shows deviations of each of the clustering types from the centralized system. The results show that the more dynamic adaptation scenarios perform better in terms of supply and demand matching, but require more frequent reconfiguration and changes in cluster topology. Thus, for the purpose of further experiments, hourly clustering type is used.

\section{Experiment set 2: Prioritization and reliability assessment}

The results in this section present reliability assessment of non-self-sufficient agents only, i.e. those agents that do not have any or enough production of their own to meet their demand (see "Prioritization" subsection). The reason for this is that the self-sufficient 


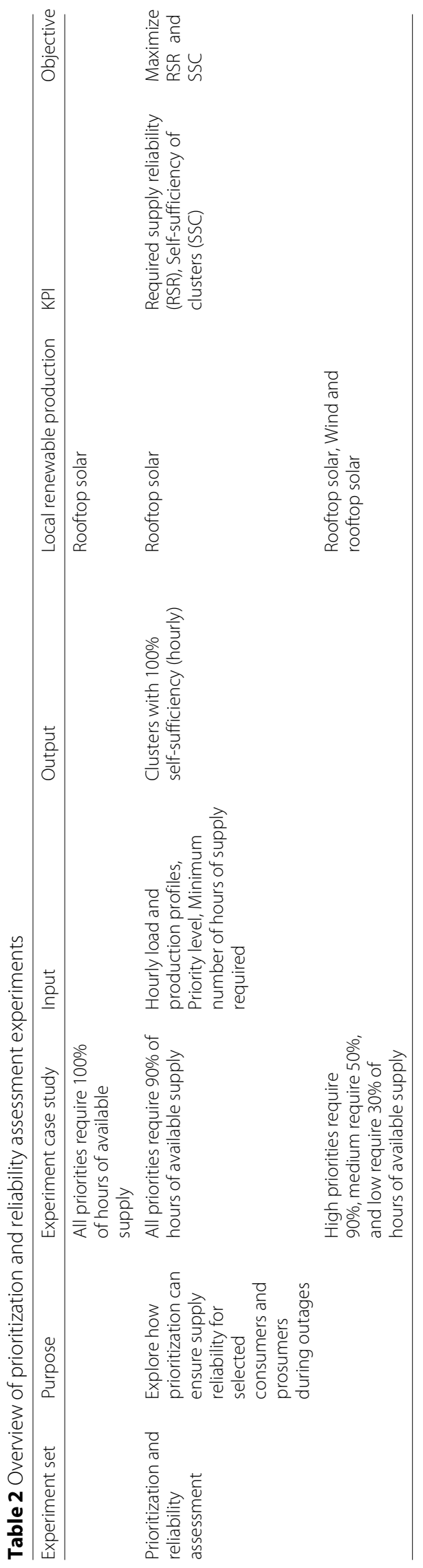




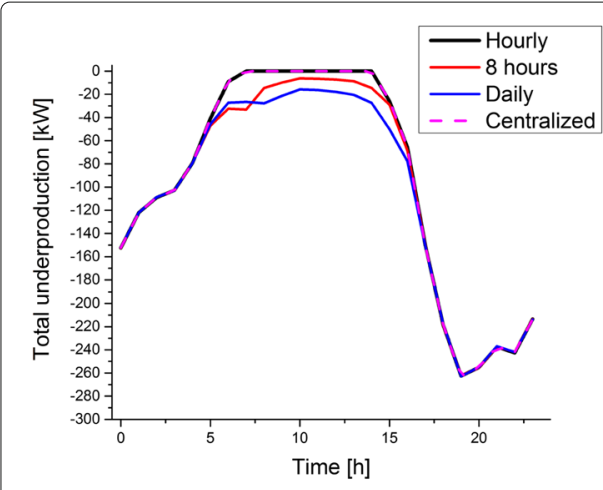

(a)

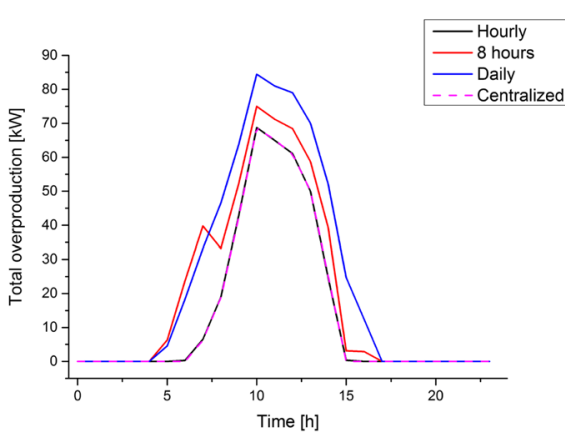

(b)

Fig. 5 Comparison of clustering mechanisms in terms of $\mathbf{a}$ total underproduction and $\mathbf{b}$ total overproduction

agents already have enough electricity available to meet their demand, and the proposed mechanism does not impact their supply reliability.

Figure 6 shows the difference between the total supply in the system (indicated by the dashed line) and the supply that is left for other consumers and prosumers, once the selfsufficient prosumers meet their own demand (indicated by the gray line). These results include rooftop solar production from prosumers only. The leftover supply is distributed to non-self-sufficient consumers and prosumers that are in the local neighborhood view of self-sufficient prosumers. This means that even after the distribution of resources, there might still be agents with unmet demand and those with (over)supply. However, their supply and demand cannot be matched because they are not in the local neighborhood of each other (i.e. they do not see each other). As can be seen, supply from self-sufficient agents is available in the system from hours 5 to 17 (inclusive), meaning that non-self-sufficient agents can receive electricity for $13 \mathrm{~h}$. However, there might not be enough electricity for all of the agents that need it, or electricity that is available might not be enough to meet the demand of any of the non-self-sufficient agents.

As stated in "Experiment set 2: Prioritization and reliability assessment" subsection, three separate case scenarios are run to observe the effect of changing the minimum required number of hours of supply on reliability assessment. The results obtained in each of the scenarios are presented in the rest of the section.

In the first case scenario, all priorities are required to be supplied during all, i.e. $100 \%$ of the hours of leftover supply. In terms of required supply reliability (RSR), results show that when all priorities require to be supplied during the entire period with supply left in the system (see Fig. 7a), only 7.69\% of high priority agents meet this requirement (see Table 4). At the same time, none of the medium and low priorities meet the requirement. The reason for the low percentage of high priorities that meet their requirement is that at hour 17 , there is not enough available supply in the system to meet the demand of all the

Table 3 Clustering type deviation from centralized system

\begin{tabular}{lll}
\hline Clustering type & Underproduction deviation from centralized & Overproduction deviation from centralized \\
\hline Hourly & $0.06 \%$ & $0.29 \%$ \\
$8 \mathrm{~h}$ & $5.97 \%$ & $40.45 \%$ \\
Daily & $10.19 \%$ & $68.95 \%$ \\
\hline
\end{tabular}




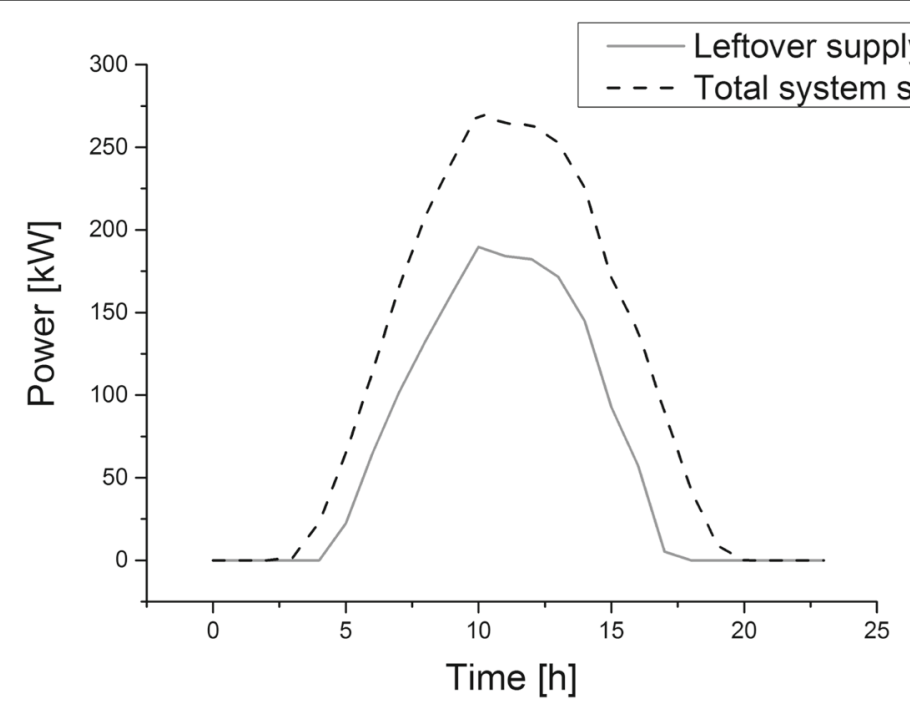

Fig. 6 Total supply in the system and leftover supply (after self-consumption by prosumers) - Solar rooftop production only

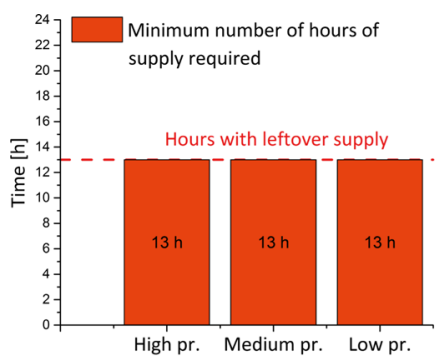

(a)

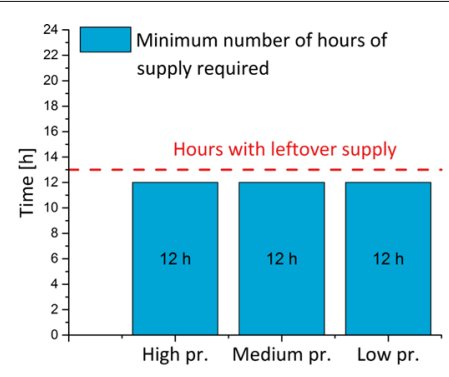

(b)

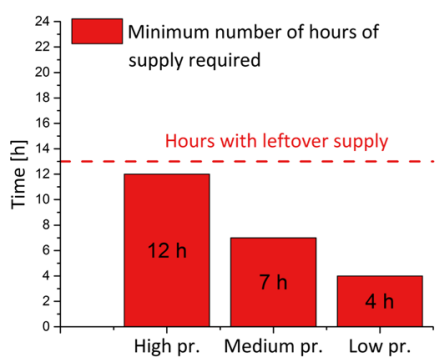

(c)

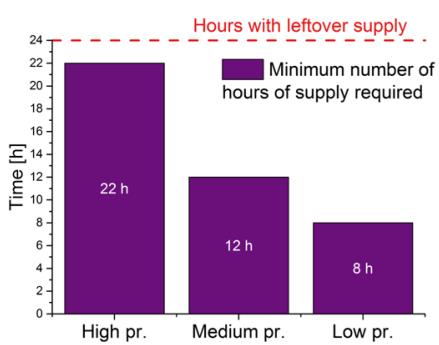

(d)

Fig. 7 Minimum required number of hours of supply. a Minimum required number of hours of supply - all priorities require 13 hours of supply, b Minimum required number of hours of supply - all priorities require 12 hours of supply, c Minimum required number of hours of supply - all priorities require a different number of hours of supply, $\mathbf{d}$ Minimum required number of hours of supply - all priorities require a different number of hours of supply - Wind and solar production 
Table 4 Required supply reliability assessment of non-self-sufficient agents - all agents need to be supplied during all the hours with leftover supply

\begin{tabular}{llll}
\hline Priority level & $\begin{array}{l}\text { Agents supplied } \\
\text { as demanded }\end{array}$ & $\begin{array}{l}\text { Minimum required } \\
\text { number of hours of supply }\end{array}$ & $\begin{array}{l}\text { Number of hours with } \\
\text { leftover supply }\end{array}$ \\
\hline High & $7.69 \%$ & 13 & 13 \\
Medium & $0 \%$ & 13 & 13 \\
Low & $0 \%$ & 13 & 13 \\
\hline
\end{tabular}

high priority agents. This is both due to high demand at that hour and only rooftop solar as the supply resource (for load and production profiles, see Fig. 2).

In the second case scenario, all priorities are required to be supplied during minimum $90 \%$ of hours of leftover supply (i.e. 12 out of $13 \mathrm{~h}$, as shown in Fig. $7 \mathrm{~b}$ ). The second set of results shows that reliability assessment is significantly impacted by adjusting the minimum required number of hours of supply. As shown in Table 5, 83.33\% of high priority agents meet the requirement, as during $12 \mathrm{~h}$ there is enough leftover supply for the majority of high priorities to be supplied. Still, however, there is not enough supply left during those hours for medium and low priorities to be supplied. Thus, the percentage of these priorities that meet their minimum requirement is $0 \%$. According to these results, $83.33 \%$ of high priority non-self-sufficient agents have reliable power supply during minimum 12 $h$ of a 24 h outage.

In the last case scenario, different priorities require supply for different minimum number of hours of leftover supply. As shown in Fig. 7c, high priorities require a minimum of $12(90 \%)$ hours of leftover supply, medium priorities require a minimum of 7 (50\%) hours of leftover supply, and low priorities require a minimum of 4 (30\%) hours of leftover supply. The highest priorities require reliable power supply during the longest period of time, while the lower priorities can only be supplied with electricity that is left after supplying high priorities. Table 6 shows that 83.33\% high priorities meet the $12 \mathrm{~h}$ minimum required for reliable supply, while $100 \%$ medium priorities meet their minimum of $7 \mathrm{~h}$, and, finally, $100 \%$ of low priorities meet the $4 \mathrm{~h}$ minimum. This means that $100 \%$ of both medium and low priorities are supplied during the period when there is supply left after the demand of high priorities is met. Looking back, Fig. 6 shows that during the day there is a solar production peak, and that peak lasts for several hours that are enough to meet the requirement of $7 \mathrm{~h}$ minimum of medium and $4 \mathrm{~h}$ minimum supply of low priorities.

Figure 8 shows the percentage of non-self-sufficient agents for each of the priorities that are in $100 \%$ self-sufficient clusters, for every hour. Self-sufficient clusters are those that rely only on their own production resources to meet the demand of all of their member agents. The clusters can have members with different priorities. As can be seen, $100 \%$ of high priorities are in $100 \%$ self-sufficient clusters during the daylight period when there is a lot of leftover supply, while there is a somewhat lower percentage during hours 4 and 17,

Table 5 Required supply reliability assessment of non-self-sufficient agents - all agents require to be supplied for $90 \%$ of the hours with leftover supply

\begin{tabular}{llll}
\hline Priority level & Agents supplied as demanded & $\begin{array}{l}\text { Minimum required } \\
\text { number of hours of supply }\end{array}$ & $\begin{array}{l}\text { Number of hours with } \\
\text { leftover supply }\end{array}$ \\
\hline High & $83.33 \%$ & 12 & 13 \\
Medium & $0 \%$ & 12 & 13 \\
Low & $0 \%$ & 12 & 13 \\
\hline
\end{tabular}


Table 6 Required supply reliability assessment of non-self-sufficient agents - all agents require to be supplied for a different number of hours with leftover supply

\begin{tabular}{llll}
\hline Priority level & Agents supplied as demanded & $\begin{array}{l}\text { Minimum required } \\
\text { number of hours of } \\
\text { supply }\end{array}$ & $\begin{array}{l}\text { Number of hours with } \\
\text { leftover supply }\end{array}$ \\
\hline High & $83.33 \%$ & 12 & 13 \\
Medium & $100 \%$ & 7 & 13 \\
Low & $100 \%$ & 4 & 13 \\
\hline
\end{tabular}

when supply starts rising and declining, respectively, and demand fluctuates significantly. Medium priorities are all supplied during the hours with high leftover supply, while the low priorities are, overall, supplied the least. Recall that the highest number of agents have low priority, followed by medium and then high priority agents. This ratio affects the percentage of low priorities in $100 \%$ self-sufficient clusters.

To add more local generation diversity, the last case scenario (when each priority requires a different minimum number of hours of supply) is run by adding wind production to the system. As stated in "Experimental setup" subsection, one intermediate-size $500 \mathrm{~kW}$ wind turbine is modeled as an additional power source in the community. Note that, due to distributed information exchange that builds of the local geographic view, the wind turbine is not in the vicinity of all of the agents. Thus, for some, solar production is still the only resource available. Figure 9 shows the difference between the total and leftover power supply in the system. Figure $7 \mathrm{~d}$ shows that, due to wind production, leftover supply is now available for $24 \mathrm{~h}$. The same scenario setup is used, with the minimum required percentage of hours of leftover supply set to $90 \%$ for high, $50 \%$ for medium, and $30 \%$ for low priorities. Thus, now, high priorities require to be supplied for minimum 22 $\mathrm{h}$, medium priorities require minimum $12 \mathrm{~h}$, while low priorities require minimum $8 \mathrm{~h}$ of leftover supply. Table 7 shows that now, $85.71 \%$ of high priority, non-self-sufficient agents are supplied by electricity during at least $22 \mathrm{~h}$ of the day, $100 \%$ of medium priorities are

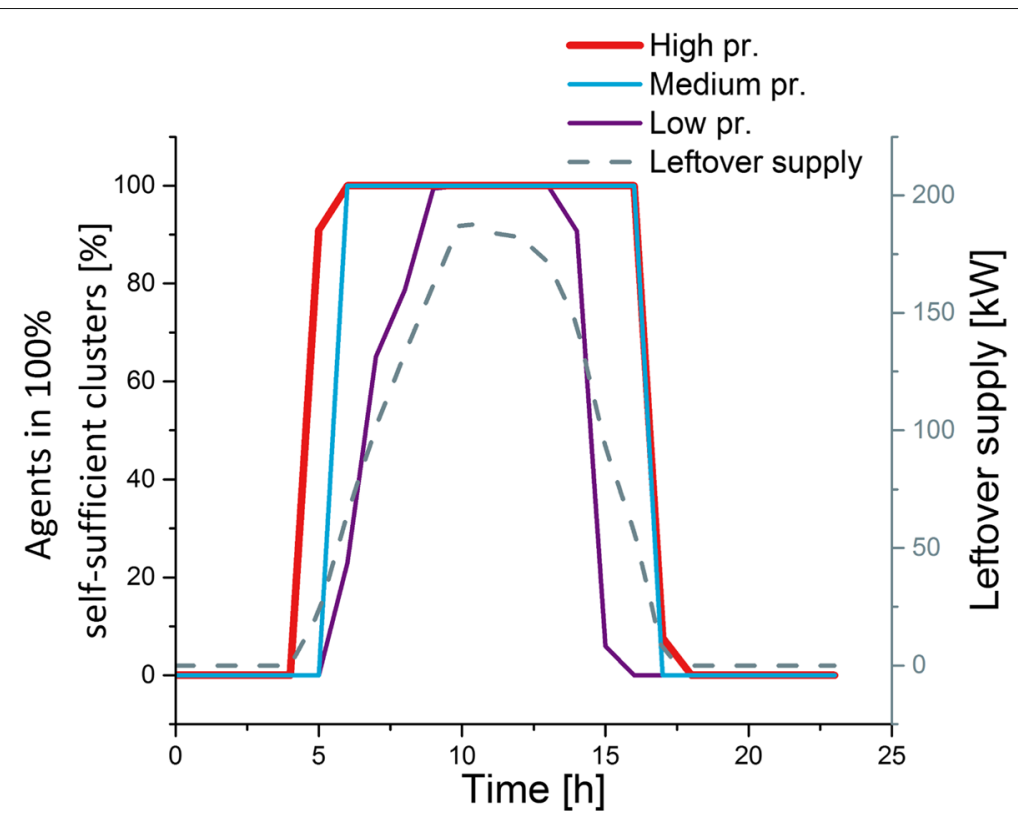

Fig. 8 Percentage of non-self-sufficient agents of each priority that are in 100\% self-sufficient clusters 


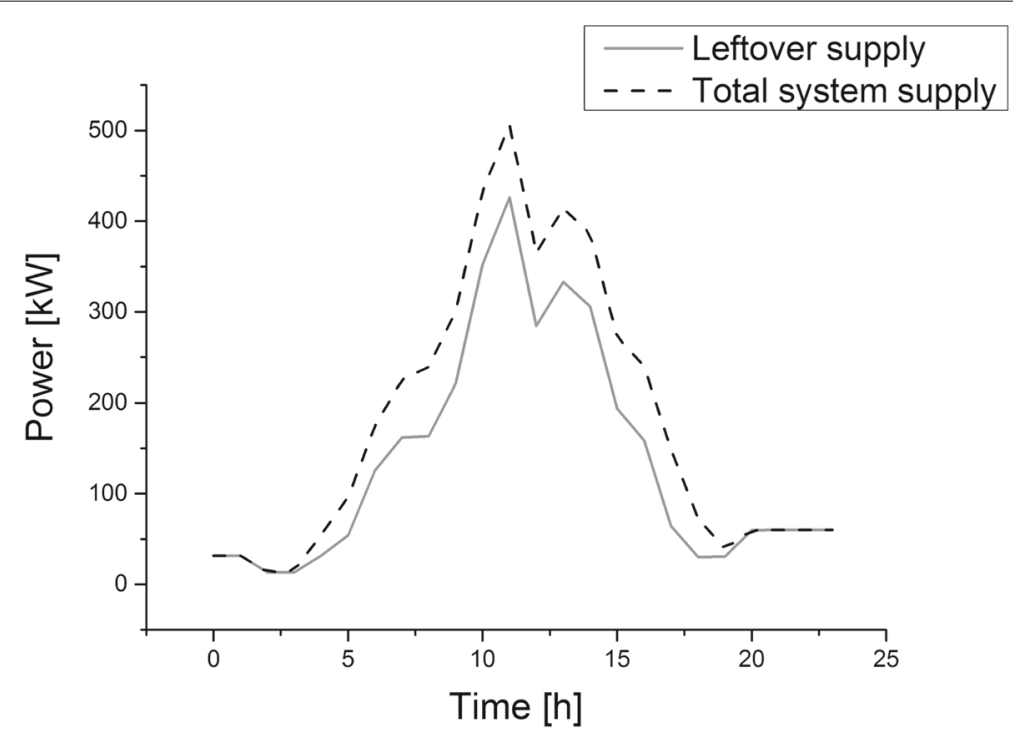

Fig. 9 Total supply in the system and leftover supply (after self-consumption by prosumers) - Wind and solar production

supplied during at least $12 \mathrm{~h}$ of the day, while $91.76 \%$ of low priorities are supplied during at least $8 \mathrm{~h}$ of the day. This difference between medium and low priorities is due to fluctuations in wind power available during the day. At intervals when there is no solar production, all consumers and prosumers are non-self-sufficient. Thus, available wind power is distributed to all of the high priorities that have the wind turbine in their local view, and the rest is distributed to medium and low priorities. Figure 10 shows that now, a high percentage of high priorities is in $100 \%$ self-sufficient clusters during the entire duration of the outage. The percentage of both medium and low priorities in $100 \%$ selfsufficient clusters fluctuates more significantly. However, due to wind production, this percentage is overall increased, compared to the case where rooftop solar production was the only available resource in the system.

As expected, the results from reliability assessment (see "Experiment set 2: Prioritization and reliability assessment" subsection) show that supply reliability primarily depends on the type of resources available, but also on the ratio between high, medium and low priorities in the system. If solar production is the only resource available, leftover supply is available during the sunny period of the day. However, if wind power generation is also present in the system, there is more flexibility, and overall more supply is available in the system. Thus, the performance of the proposed mechanism in terms of reliability improvement is fully-dependent on the type of local resources available in an impacted area. Given only solar and wind generation are available, the results would significantly

Table 7 Required supply reliability assessment of non-self-sufficient - all agents require to be supplied for a different number of hours with leftover supply - Wind and solar production)

\begin{tabular}{llll}
\hline Priority level & Agents supplied as demanded & $\begin{array}{l}\text { Minimum required } \\
\text { number of hours of supply }\end{array}$ & $\begin{array}{l}\text { Number of hours with } \\
\text { leftover supply }\end{array}$ \\
\hline High & $85.71 \%$ & 22 & 24 \\
Medium & $100 \%$ & 12 & 24 \\
Low & $91.76 \%$ & 8 & 24 \\
\hline
\end{tabular}




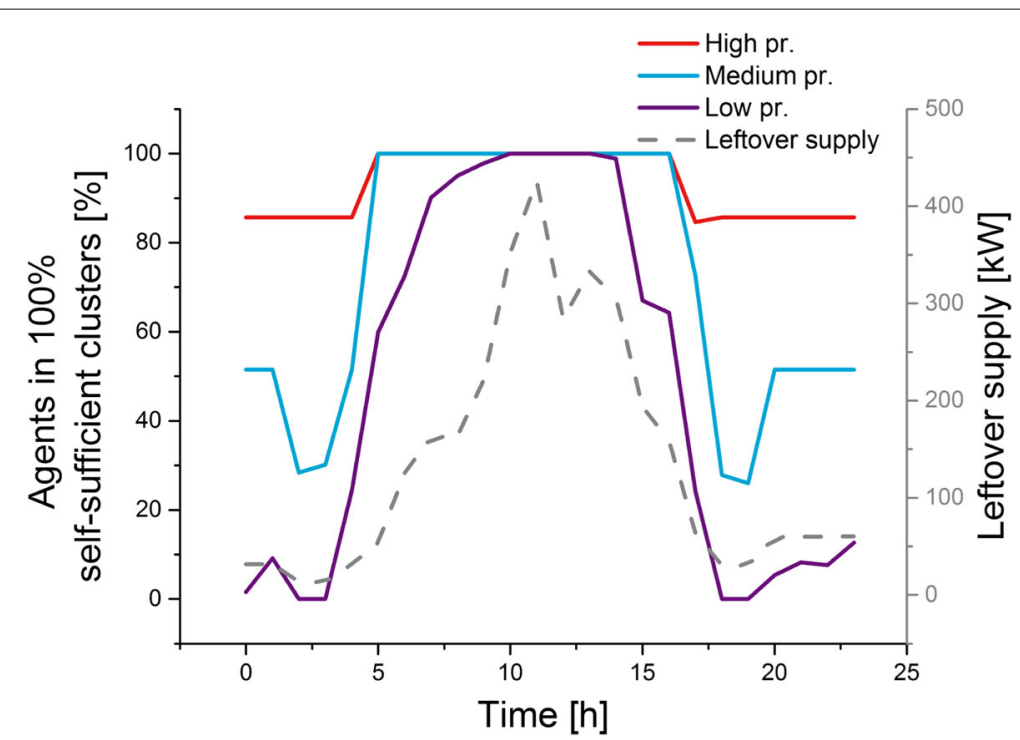

Fig. 10 Percentage of non-self-sufficient agents of each priority that are in 100\% self-sufficient clusters Wind and solar production

differ on a windless, cloudy day. As an alternative, other types of resources (e.g. storage) can be used for different periods.

The reliability assessment itself depends on several parameters, including setting of the minimum required number of hours of leftover supply for every priority, as well as the ratio between high, medium and low priorities in the system. The required minimum number of hours of leftover supply depends on the supply availability, types of resources in the system and the knowledge of agents. In practice, a more extensive study would be needed to set parameters appropriately for specific situations.

\section{Discussion}

The results discussed in the previous section show that the mechanism proposed in this paper can be used as an ICT platform that enables decentralized supply and demand matching in changing environments. Decentralized supply and demand matching can be valuable if and when there is no central information on total and individual supply and demand in the system, and the backbone grid is unavailable. By facilitating local energy sharing, prolonged power supply is ensured for members of communities affected by an outage. Thus, a functioning mechanism is developed that improves supply reliability by maximizing local resource utilization.

Besides providing a functioning platform for local energy exchange during outages, the possible applications of the mechanism are many. As a tool, it can be used by decision and policy makers to run extensive scenarios for specific communities, demonstrating what impact community's decisions regarding consumer prioritization have on the level of its supply reliability. These case scenarios can help explain different implications of, for example, varying the percentage of high, medium and low priorities, or fixing the priorities for selected consumers. This, in turn, can be used to make decisions on additional resources needed to achieve reliability goals of specific communities. This can include 
installing generators, additional solar panels or (community) energy storage. As the mechanism is highly adaptable, it provides potential for resilient energy communities that dynamically adapt to changes to respond to rapid onset events (Tierney and Bruneau 2007; Adil and Ko 2016; Arghandeh et al. 2014; Jiménez-Estévez et al. 2017).

More responsible resource planning by, for example, avoiding curtailing of overproduction by sharing energy or installing community-owned storage based on the specific community's needs, promotes more sustainable use of resources, especially when resources are scarce. Ultimately, relying on local production only can lead to less dependence on the main grid, even when the backbone grid is available, increasing self-sufficiency of local (energy) communities.

The application of the proposed mechanism can potentially have wider implications in terms of social interaction and cohesion in impacted communities. Instrumental to the mechanism is the consumer differentiation as a means to determine how to distribute electricity according to a community's preferences. Here, the basis for differentiation, i.e. priorities, are determined by the community members themselves, based on their perceived social values. In this paper, the differentiation process is not automated; the power of deciding on priorities is given to the community members, which means that they have to come together and reach a mutual agreement on consumer priorities. By giving them a common goal, such as increasing supply reliability during outages, the mechanism enforces social interaction of different stakeholders, which can ultimately lead to social cohesion (Šćepanović et al. 2017; Alvial-Palavicino et al. 2011; Ubilla et al. 2014; Arentsen and Bellekom 2014). Thus, community members are empowered to make decisions that directly benefit community members. Even though social cohesion is one of the possible outcomes, depending on the community, if misused such a system could also possibly have negative implications towards more vulnerable groups. More research is needed to determine this. However, the study of these implications is outside the scope of this paper.

Alternatively, assigning priorities can also be done by institutions (e.g. distribution system operators), in cooperation with the local communities, depending on the specific situation or a type of consumer or prosumer. The process of assigning priorities can also differ based on the type of outage, e.g. whether it is planned or unplanned. Finally, priorities can be static (fixed) or dynamic, adapting to changing circumstances and specific community's preferences, depending on the time of the outage for example.

\section{Conclusions}

This paper proposes a mechanism that enables decentralized supply and demand matching, based only on locally available information and self-organization of consumers and prosumers. The proposed mechanism empowers local communities to decide for themselves how local resources are distributed during events such as outages, ensuring prolonged power supply for differentiated members of affected communities. In addition the paper shows that more frequent reconfiguration scenarios generate the overall lowest total overproduction and underproduction, and deviate the least from the centralized approach.

On a higher level, this paper shows that decision making between individual consumers and prosumers can benefit the affected communities by sharing available locally produced energy, given appropriate mechanisms. In case of scheduled outages, the mechanism can 
be used by decision makers and planners beforehand as a tool to gain an insight how different community perspectives on energy priorities can influence supply reliability in affected communities. Eventually, based on the results, backup plans for alternative power sources can be made.

Negotiation mechanisms for agreeing upon resource sharing between different consumers and prosumers will be explored in detail in the future work. Current work uses the mechanism proposed in this paper in combination with distribution system reconfiguration to assess if power systems can be made more resilient in case of large-scale outage caused by a rapid onset event.

The future brings challenges. To face these challenges, power systems will need new paradigms for coordination and planing. Harnessing the potential of renewable resources and smart technologies for intelligent coordination through empowerment of consumers to become pro-active participants is a promising solution for the future power systems.

\section{Acknowledgements}

Not applicable.

\section{Authors' contributions}

SČ, MW and FB determined the scope of the research, the research questions to be addressed, discussed the results, outlined the paper and contributions. SČ and MW designed the experiments, discussed and drafted the results. Š̌ developed the mechanisms, collected the data, conducted the experiments and drafted versions of the paper (in close interaction with MW). MW and FB edited different versions of the paper.

\section{Funding}

This work is funded by the NWO project Adaptive clustering for Decentralised Resilient Energy Management (ADREM), grant number 629.002.002

\section{Availability of data and materials}

The datasets analyzed during the current study are publicly available by NEDU and Koninklijk Nederlands Meteorologisch Institut (KNMI) repository, http://nedu.nl/portfolio/verbruiksprofielen/ and http://projects.knmi.nl/klimatologie/ uurgegevens/selectie.cgi

The results data and the simulation tool are available from the corresponding author on reasonable request.

\section{Competing interests}

The authors declare that they have no competing interests.

Received: 1 April 2019 Accepted: 29 May 2019

Published online: 02 July 2019

\section{References}

Adil AM, Ko Y (2016) Socio-technical evolution of Decentralized Energy Systems: A critical review and implications for urban planning and policy. Renew Sustain Energy Rev 57:1025-1037. [Online]. Available: http://dx.doi.org/10.1016/j. rser.2015.12.079

Adu-Kankam KO, L. M. -Matos LM (2018) Towards Collaborative Virtual Power Plants. Sustain Energy, Grids Netw 16:28-39. [Online]. Available: http://link.springer.com/10.1007/978-3-319-78574-5_3

Alvial-Palavicino C, Garrido-Echeverría N, Jiménez-Estévez G, Reyes L, Palma-Behnke R (2011) A methodology for community engagement in the introduction of renewable based smart microgrid. Energy Sustain Dev 15(3):314-323. [Online]. Available: http://dx.doi.org/10.1016/j.esd.2011.06.007

Amin M (2001) Toward self-healing energy infrastructure systems. Comput Appl Power, IEEE 14(1):20-28

Amin M, Stringer J (2008) The Electric Power Grid: Today and Tomorrow. MRS Bull 33(04):399-407

Arentsen M, Bellekom S (2014) Power to the people : local energy initiatives as seedbeds of innovation ?. Energy Sustain Society:1-12

Arghandeh R, Brown M, Del Rosso A, Ghatikar G, Stewart E, Vojdani A, Von Meier A (2014) The local team: Leveraging distributed resources to improve resilience. IEEE Power Energy Mag 12(5):76-83

Asmus P (2010) Microgrids, Virtual Power Plants and Our Distributed Energy Future. Electr J 23(10):72-82. [Online]. Available: http://dx.doi.org/10.1016/j.tej.2010.11.001

Brazier FMT, Poutré H, Abhynakar AR, Saxena K, Singh SN, Tomar KK (2015) A Review of Multi Agent Based Decentralised Energy Management Issues. In: Proceedings of the 2nd international conference on energy and environment: Bringing together engineering and economics. IEEE Society. pp 1-5

Brooklyn Microgrid (2019). https://www.brooklyn.energy/. Accessed 06 Jun 2019

Cai Y, Huang T, Bompard E, Cao Y, Li Y (2017) Self-Sustainable Community of Electricity Prosumers in the Emerging Distribution System. IEEE Trans Smart Grid 8(5):2207-2216

Čaušević S, Warnier M, Brazier FMT (2017) Dynamic, self-organized clusters as a means to supply and demand matching in large-scale energy systems. Proc 14th IEEE Int Conf Netw Sens Control. (To appear)

Clark KP (2014) Negotiation and Monitoring in Open Environments. Dissertation, Delft University of Technology

Duckham M (2013) Decentralized Spatial Computing. 1st. Springer-Verlag, Berlin 
EWT DW52 500kW. https://cdn2.hubspot.net/hubfs/4248001/Downloads/Brochures/EN/EWT_Flyer-dw52-500kW.pdf. Accessed 06 Jun 2019.

Farhangi $\mathrm{H}$ (2010) The path of the smart grid. IEEE Power Energy Mag 8(1):18-28

Farzin H, Member S, Fotuhi-firuzabad M (2016) Enhancing Power System Resilience Through Hierarchical Outage Management in Multi-Microgrids. IEEE Trans Smart Grid 7(6):2869-2879

Gao H, Chen Y, Xu Y, Liu C (2016) Resilience-oriented critical load restoration using microgrids in distribution systems. IEEE Trans Smart Grid 7(6):2837-2848

Grosse C, Olausson P (2018) Swedish multi-level planning system for critical infrastructure protection : The regional core. In: Proceedings of ESREL 2018, June 17-21, 2018, Trondheim, Norway. pp 1893-1901

Hatziargyriou N, Asano H, Iravani R, Marnay C (2007) Microgrids. IEEE Power Energy Mag 5(4):78-94

Huang X, Yang Y, Taylor GA (2016) Service restoration of distribution systems under distributed generation scenarios. CSEE J Power Energy Syst 2(3):43-50

Hufen JAM, Koppenjan JFM (2015) Local renewable energy cooperatives : revolution in disguise ?. Energy Sustain Society. [Online]. Available: http://dx.doi.org/10.1186/s13705-015-0046-8

Ilic MD, Xie L, Khan UA, Moura JMF (2010) Modeling of future cyber-physical energy systems for distributed sensing and control. IEEE Trans Syst Man Cybern Syst Hum 40(4):825-838

Jelasity M, Montresor A, Babaoglu O (2005) Gossip-based aggregation in large dynamic networks. ACM Trans Comput Syst 23(3):219-252

Jiménez-Estévez G, Navarro-Espinosa A, Palma-Behnke R, Lanuzza L, Velázquez N (2017) Achieving resilience at distribution level: Learning from isolated community microgrids. IEEE Power Energy Magaz 15(3):64-73

Jung O, Bessler S, Ceccarelli A, Zoppi T, Vasnev A, Montoya L, Clarke T, Chappell K (2016) Towards a Collaborative Framework to Improve Urban Grid Resilience. In: Energy Conference (ENERGYCON), 2016 IEEE International

Katre A, Tozzi A, Bhattacharyya S (2019) Sustainability of community-owned mini- grids : evidence from India. Energy Sustain Society 9

Koninklijk Nederlands Meteorologisch Instituut. Uurgegevens van het weer in Nederland. http://projects.knmi.nl/ klimatologie/uurgegevens/selectie.cgi. Accessed 25 Aug 2017.

Landegren F, Johansson J, Samuelsson O (2014) Comparing societal consequence measures of outages in electrical distribution systems(T. Nowakowski, M. Mlynczak, A. Jodejko-Pietruczuk, S. Webinska-Wojciechowska, eds.)

Lemmens J, van der Burgt J, Bosma T, van den Wijngaart, van Bemmel B, Koelemeijer R (2014) Het potentieel van zonnestroom in de gebouwde omgeving van Nederland. Planbureau voor de Leefomgeving, Tech. Rep. https:// www.pbl.nl/sites/default/files/cms/publicaties/pbl-2014-dnv-gl-hetpotentieel-van-zonnestroom-in-de-gebouwdeomgeving-van-nederland_01400.pdf. Accessed 10 Jun 2017

Li Z, Shahidehpour M, Aminifar F, Alabdulwahab A, Al-Turki Y (2017) Networked Microgrids for Enhancing the Power System Resilience. Proceedings of the IEEE 105(7):1289-1310

Matasci S (2017) What is the average solar panel size and weight?. [Online]. Available: http://news.energysage.com/ average-solar-panel-size-weight/

Methenitis G, Kaisers M, La Poutre H (2018) Renewable electricity trading through SLAs. Energy Inform 1:1-17

Mondal A, Illindala MS, Khalsa AS, Klapp DA, Eto JH (2016) Design and operation of smart loads to prevent stalling in a microgrid. IEEE Trans Ind Appl 52(2):1184-1192

NEDU - Verbruiksprofielen. https://www.nedu.nl/documenten/verbruiksprofielen/. Accessed 16 Aug 2017

Oteman M, Wiering M, Helderman J-k (2014) The institutional space of community initiatives for renewable energy : a comparative case study of the Netherlands, Germany and Denmark. Energy Sustain Society:1-17

OVO Energy (2014) White paper: community energy. Technical report, OVO Energy, Bristol. https://www.ovoenergy.com/ binaries/content/assets/documents/pdfs/community_energy_whitepaper.pdf. Accessed 10 Nov 2018.

Palensky P, Widl E, Elsheikh A (2014) Simulating Cyber-Physical Energy Systems: Challenges, Tools and Methods. Trans Syst Man Cybern Syst 44(3):318-326

Panteli M, Mancarella P (2015) The Grid: Stronger, Bigger, Smarter?. IEEE Power Energy Magaz April

Patel MR (1999) Wind and Solar Power Systems. CRC Press LLC, Boca Raton

Reddy GH, Chakrapani P, Goswami AK, Choudhury NBD (2017) Prioritization of Load Points in Distribution System considering Multiple Load Types using Fuzzy Theory. In: 2017 IEEE International Conference on Fuzzy Systems (FUZZ-IEEE). IEEE. pp 1-6

Šćepanović S, Warnier M, Nurminen JK (2017) The role of context in residential energy interventions : A meta review. Renew Sustain Energy Rev 77(May):1146-1168

Sheldrick A, Tsukimori O (2017) Quiet energy revolution underway in Japan as dozens of towns go off the grid. [Online]. Available: https://www.reuters.com/article/us-japan-energy-revolution/quiet-energy-revolution-underway-injapan-as-dozens-of-towns-go-off-the-grid-idUSKCN1BUOUT

Silva R, Ferreira A, Ferreira A, Leitao P (2015) Increasing self-sustainability in micro grids using load prioritization and forecasting mechanisms. In: IEEE 10th Conference on Industrial Electronics and Applications (ICIEA). IEEE. pp 1069-1074

Strbac G, Hatziargyriou N, Lopes JP, Moreira C, Dimeas A, Papadaskalopoulos D (2015) Microgrids: Enhancing the Resilience of the European Megagrid. IEEE Power Energy Mag 13(3):35-43

Taha AF, Panchal JH (2014) Multilevel Decision-making in Decentralized Energy Systems with Multiple Technologies and Uncertainty. IEEE Trans Syst Man Cybern Systems 44(7):894-907

Tierney K, Bruneau M (2007) A Key to Disaster Loss Reduction. TR News:14-18. [Online]. Available: http://onlinepubs.trb. org/onlinepubs/trnews/trnews250_p14-17.pdf

Tushar W, Zhang JA, Smith DB, Poor HV, Thiebaux S (2014) Prioritizing consumers in smart grid: A game theoretic approach. IEEE Trans Smart Grid 5(3):1429-1438

Wollenberg BFSMA (2005) Toward smart grid. Power october:34-41

Wood AJ, Wollenberg BF, Sheble GB (2013) Power Generation, Operation and Control 3rd ed. Wiley-Interscience

Ubilla K, Jiménez-estévez GA, Member S, Hernádez R, Reyes-chamorro L, Member S, rrigoyen CH, Severino B, Palma-behnke R, Member S (2014) Smart Microgrids as a Solution for Rural Electri fi cation : Ensuring Long-Term Sustainability Through Cadastre and Business Models. IEEE Trans Sustain Energy 5(4):1310-1318

\section{Publisher's Note}

Springer Nature remains neutral with regard to jurisdictional claims in published maps and institutional affiliations. 